\title{
A NEW TECHNIQUE FOR HIGH-RESOLUTION FREQUENCY DOMAIN OPTICAL COHERENCE TOMOGRAPHY
}

\author{
S. Chandra Sekhar ${ }^{1}$, Himanshu Nazkani ${ }^{2}$, Thierry Blu ${ }^{3}$, Michael Unser ${ }^{4}$ \\ Biomedical Imaging Laboratory, Institute of Imaging and Applied Optics \\ École Polytechnique Fédérale de Lausanne, Lausanne, Switzerland, CH-1015. \\ Email: $\left\{{ }^{1}\right.$ chandrasekhar.seelamantula, ${ }^{2}$ himanshu.nazkani, ${ }^{3}$ thierry.blu, ${ }^{4}$ michael.unser $\} @$ epfl.ch
}

\begin{abstract}
Frequency domain optical coherence tomography (FDOCT) is a new technique that is well-suited for fast imaging of biological specimens, as well as non-biological objects. The measurements are in the frequency domain, and the objective is to retrieve an artifact-free spatial domain description of the specimen. In this paper, we develop a new technique for model-based retrieval of spatial domain data from the frequency domain data. We use a piecewise-constant model for the refractive index profile that is suitable for multi-layered specimens. We show that the estimation of the layered structure parameters can be mapped into a harmonic retrieval problem, which enables us to use high-resolution spectrum estimation techniques. The new technique that we propose is efficient and requires few measurements. We also analyze the effect of additive measurement noise on the algorithm performance. The experimental results show that the technique gives highly accurate parameter estimates. For example, at $25 \mathrm{~dB}$ signal-to-noise ratio, the mean square error in the position estimate is about $0.01 \%$ of the actual value.
\end{abstract}

Index Terms : frequency domain optical coherence tomography, harmonic retrieval, annihilating filter, high-resolution method.

\section{INTRODUCTION}

Optical coherence tomography (OCT) is becoming an increasingly popular tool for imaging the three-dimensional structure of layered biological as well as non-biological specimens. FDOCT is a recent alternative to the already established time domain OCT (TDOCT). FDOCT systems have two advantages: (1) They are much faster than TDOCT because the acquisition is done without scanning and (2) They offer greater sensitivity with a detection threshold well above $80 \mathrm{~dB}$, even in situations with low light levels [1]. The first medical application of FDOCT is described in [2], and the first in-vivo images are reported in $[3,4]$. The FDOCT measurements are in the frequency domain and the conventional reconstruction approach is to apply an inverse Fourier transform. However, this

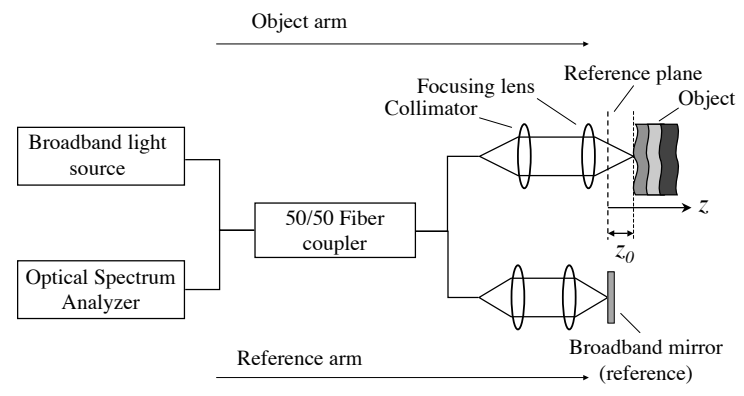

Fig. 1. Schematic of the FDOCT system experimental setup

technique has shortcomings and is known to introduce artifacts [5]. Also, it is not suitable if we are interested in accurate estimates of the specimen parameters. Given the nascency of the field, not much research has gone into the signal processing aspects of the technique. The most recent result in this direction is the use of minimum-phase functions to develop a Fienup-like iterative reconstruction algorithm [6].

In this paper, we take a model-based approach and develop a new technique for accurate parameter retrieval from the FDOCT measurements.

\section{FDOCT SIGNAL ACQUISITION}

The FDOCT experimental setup is shown in Fig.1. The output of a broadband light source is split into two beams. Each beam is directed towards one arm of a Michelson interferometer. The light reflected from the broadband mirror serves as the reference signal. The reference and the light reflected from the object are combined in a collinear fashion by the fiber coupler and directed towards an optical spectrum analyzer. The signal from the object consists of many elementary waves emanating from different depths $z$ within the object. The measured interference signal $I(\nu)$ is given by:

$$
I(\nu)=S(\nu)\left|a_{R} e^{j 2 k r}+\int_{0}^{\infty} b(z) e^{j 2 k[r+n(z) z]} d z\right|^{2}
$$


where,

$k=$ wave number; $k=\frac{2 \pi}{\lambda}=\frac{2 \pi \nu}{c}$, where $\lambda$ is the wavelength, $\nu$ is the frequency and $c$ is the speed of light in vacuum.

$2 r=$ pathlength in the reference arm,

$2(r+z)=$ pathlength in the object arm

$2 z=$ pathlength in the object arm measured from the reference plane

$z_{0}=$ offset distance between the reference plane and the object surface

$n(z)=$ refractive index as a function of the depth $z$

$a_{R}=$ reference wave amplitude

$b(z)=$ amplitude of the wave backscattered from the object

$S(\nu)=$ spectral intensity distribution of the broadband light source

Without loss of generality, we set $a_{R}=1$ and rewrite (1) as:

$$
I(\nu)=S(\nu)\left|1+\int_{0}^{\infty} b(z) e^{j 2 k n(z) z} d z\right|^{2}
$$

From the intensity measurements, one can extract the pathlength differences, which is the information that we are interested in. Specifically, given $I(\nu)$, we want to recover $b(z)$. This is the fundamental inverse problem in FDOCT. The term $B(\nu)=\int_{0}^{\infty} b(z) e^{j 2 k n(z) z} d z$ is the frequency response of the specimen. Therefore, we have: $I(\nu)=S(\nu)|1+B(\nu)|^{2}$ with $B(\nu)$ unknown, which is a non-linear estimation problem.

\section{THE MULTILAYER SPECIMEN MODEL}

We model the specimen as a concatenation of layers, each having a fixed refractive index. Thus, we have a piecewiseconstant refractive index profile $n(z)$. The light propagating through the specimen gets reflected at the interfaces because of the change in refractive index. The reflected light intensity therefore can be written as a sum of Dirac-delta impulses, each impulse corresponding to a discontinuity in the refractive index profile. This constitutes our model of the impulse response of the object, whose unknown parameters are the positions $b_{\ell}$ and the weights $a_{\ell}$ of the Dirac impulses. In the Fourier domain, the problem now reduces to the following: Fit the frequency domain intensity measurements with a function of the form:

$$
I(\nu)=S(\nu)\left|1+\sum_{\ell=0}^{L} a_{\ell} e^{i 2 \pi b_{\ell} \nu}\right|^{2}
$$

by making an appropriate choice of the parameters $\left\{a_{\ell}, b_{\ell}, \ell=\right.$ $0,1,2, \ldots, L\}$. In terms of the notation that we introduced in Sec. 2, we have $B(\nu)=\sum_{\ell=0}^{L} a_{\ell} e^{i 2 \pi b_{\ell} \nu}$. The source power spectrum $S(\nu)$ can either be measured by blocking the object arm fully or estimated by averaging over an ensemble of independent lateral scans or measurements. Thus, we can assume that it is known a priori.

Our objective is to estimate the parameters $a_{\ell}$ and $b_{\ell}$ as accurately as possible. This will enable us to compute the specimen parameters such as the refractive index and the thickness of each layer accurately.

\section{THE INVERSE PROBLEM AND HARMONIC RETRIEVAL}

We consider the observations $I(\nu)$, normalized with respect to the source power spectrum $S(\nu)$. Let $M(\nu)$ denote the normalized observations. We have

$$
\begin{aligned}
M(\nu)= & 1+\sum_{\ell=0}^{L} a_{\ell}^{*} e^{-i 2 \pi b_{\ell} \nu}+\sum_{\ell=0}^{L} a_{\ell} e^{i 2 \pi b_{\ell} \nu} \\
& +\sum_{m=0}^{L} \sum_{\ell=0}^{L} a_{\ell} a_{m}^{*} e^{-i 2 \pi\left(b_{\ell}-b_{m}\right) \nu}
\end{aligned}
$$

An important practical aspect that we need to keep in mind is the fact that the intensity of the reflected light is much smaller than that of the incident light. Stated mathematically, we have $\left|\sum_{\ell=0}^{L} a_{\ell} e^{i 2 \pi b_{\ell} \nu}\right| \ll 1$. In this paper, we refer to this as the source dominance condition. This condition also implies that $|B(\nu)|^{2}$ is very small compared to unity. We can thus write $M(\nu)$ as: $M(\nu)=1+D(\nu)+|B(\nu)|^{2}$ where $D(\nu)$ is given by

$$
D(\nu)=\sum_{\ell=0}^{L} a_{\ell} e^{i 2 \pi b_{\ell} \nu}+\sum_{\ell=0}^{L} a_{\ell}^{*} e^{-i 2 \pi b_{\ell} \nu}
$$

Now, let us consider the inverse Fourier transform of $D(\nu)$ :

$$
\mathcal{F}^{-1}\{D(\nu)\}=\sum_{\ell=0}^{L} a_{\ell} \delta\left(z+b_{\ell}\right)+\sum_{\ell=0}^{L} a_{\ell}^{*} \delta\left(z-b_{\ell}\right)
$$

We see that (6) represents a sum of Dirac impulses and we are interested in finding the strength and position of the Dirac impulses. This is similar to the problem of harmonic retrieval [7] and finite rate of innovation sampling [8]. Thanks to our model of the specimen, we are now in a perfect position to deploy the powerful annihilating filter technique [7] that is the main ingredient of all high-resolution techniques [7, 8]. If the source spectrum is Gaussian, the inverse Fourier transform of $S(\nu) D(\nu)$ will consist of a sum of spatially displaced Gaussians. This is again similar to the problem addressed in Sec.IV B of [8]. In the following sections, we present the sequential, two-step technique to compute $b_{\ell}$ and $a_{\ell}$, successively, from the samples of $D(\nu)$. In practice, we have the 
samples of $M(\nu)$. However, this is not a problem because the constant unity (see (4)) only acts as a bias/offset and the term $|B(\nu)|^{2}$ is too small in magnitude to dominate in the subspace based estimation techniques that will be presented in Sec.5.

\subsection{Solution for $b_{\ell}$}

In practice, we have only access to sampled data. The samples of $D(\nu)$ are denoted by $D_{k}$ and are assumed to be alias-free. Thus, our model takes the form:

$D_{k}=\sum_{\ell=0}^{L} a_{\ell} \gamma_{\ell}^{k}+\sum_{\ell=0}^{L} a_{\ell}^{*} \gamma_{\ell}^{* k}, k=k_{1}, k_{1}+1, \ldots, k_{1}+K-1$

where $\gamma_{\ell}=e^{i 2 \pi b_{\ell}}$. To simplify the notation, we assume that $k_{1}=0$, keeping in mind that the same analysis holds for any non-zero integer value. We define a polynomial $P(\gamma)$ with roots $\left\{\gamma_{\ell}, \gamma_{\ell}^{*}\right\}$ such that,

$$
P(\gamma)=\prod_{\ell=0}^{L}\left(\gamma-\gamma_{\ell}\right)\left(\gamma-\gamma_{\ell}^{*}\right)=\sum_{i=0}^{2 L} p_{i} \gamma^{i}
$$

where the $p_{i}$ are unknown, as yet. We can compute them from $D_{k}$ by using the annihilation property: $\sum_{i=0}^{2 L} p_{i} D_{k-i}=$ $0, \forall k \in[2 L+1, K-1]$. These linear equations are written in a matrix form as:

$$
\underbrace{\left[\begin{array}{cccc}
D_{2 L+1} & D_{2 L} & \cdots & D_{1} \\
D_{2 L+2} & D_{2 L+1} & \cdots & D_{2} \\
D_{2 L+3} & D_{2 L+2} & \cdots & D_{3} \\
\vdots & \vdots & \ddots & \vdots \\
D_{K-1} & D_{K-2} & \cdots & D_{K-1-2 L}
\end{array}\right]}_{\mathbb{D}} \underbrace{\left[\begin{array}{c}
p_{0} \\
p_{1} \\
p_{2} \\
\vdots \\
p_{2 L}
\end{array}\right]}_{\mathbf{p}}=\mathbf{0}
$$

where $\mathbf{0}$ is the null vector. In estimation theory, $\mathbb{D}$ is called the measurement matrix. If the Dirac locations are distinct and $K \geq 4 L+4$, then the equation $\mathbb{D} \mathbf{p}=\mathbf{0}$ has a unique solution up to a scale factor. The entries in $\mathbf{p}$ are the coefficients of the polynomial $P(\gamma)$. We apply a root-solver to compute $\left\{\gamma_{\ell}, \gamma_{\ell}^{*}, \ell=0,1,2, \ldots, L\right\}$ and then deduce $b_{\ell}$.

\subsection{Solution for $a_{\ell}$}

Interestingly, the previous solution for $b_{\ell}$ is independent of $a_{\ell}$ and so their values can be determined before hand. We are now faced with a much simpler linear problem. Specifically, using the pre-computed values of $\gamma_{\ell}$, we can rewrite (7) as:

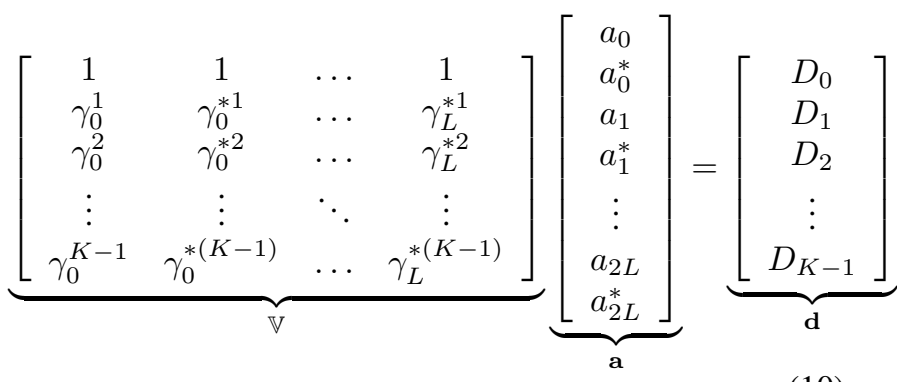

and solve this Vandermonde system of equations to obtain a. Alternatively, we can also estimate $a_{\ell}$ directly from the spectrum of $D(\nu)$. This can also be done efficiently by using the Goertzel algorithm. This alternative is also useful to circumvent matrix instability problems if $K$ is large.

In principle, any set of $4 L+4$ measurements of $D_{k}$ suffice to compute the $2 L+2$-complex valued parameters $\left\{a_{\ell}, b_{\ell}\right\}$. In practice, it is useful to introduce some redundancy to obtain a more robust scheme and to minimize the effect of noise.

\section{EFFECT OF ADDITIVE NOISE}

We also investigate the effect of measurement noise which is inevitable in a real-world experiment. The noisy measurements are given by:

$$
I(\nu)=S(\nu)\left|1+\sum_{\ell=0}^{L} a_{\ell} e^{i 2 \pi b_{\ell} \nu}\right|^{2}+N(\nu)
$$

where $N(\nu)$ denotes the spectrum of noise. In the presence of noise, we do not have exact solutions to the equations (9) and (10). However, we can solve them in the minimumnorm sense. The optimum vector $\mathbf{p}^{*}$ is defined as: $\mathbf{p}^{*}=$ $\arg \min _{\mathbf{p}}\|\mathbb{D} \mathbf{p}\|^{2}$. $\mathbf{p}^{*}$ turns out to be the minimum eigenvector of $\mathbb{D}^{T} \mathbb{D}$. Similarly, the optimum vector $\mathbf{a}^{*}$ is defined as: $\arg \min _{\mathbf{a}}\|\mathbb{V} \mathbf{a}-\mathbf{d}\|^{2}$. This yields the Moore-Penrose solution: $\mathbf{a}^{*}=\left(\mathbb{V}^{T} \mathbb{V}\right)^{-1} \mathbb{V}^{T} \mathbf{d}$.

One may also denoise the signal based on the singularvalue decomposition of $\mathbb{D}^{T} \mathbb{D}$. In practice, we compute the matrix $\mathbb{D}$ from the measurements $I(\nu)$ normalized by the measured source power spectrum. The dominant singular values correspond to the signal and the associated singular vectors span the desired signal subspace. The smaller singular values correspond to the noise and the weak signal cross-terms coming from $|B(\nu)|^{2}$. The corresponding singular vectors span a subspace that is orthogonal to the desired signal subspace.

In our analysis, we have assumed a priori knowledge of $L$, which may not always be available in practice. Many techniques exist for estimating $L$, under the popular title of orderestimation [7].

\section{SIMULATION RESULTS}

We generated experimental data for a two-layer specimen with thickness $119 \mathrm{~nm}$ and $92 \mathrm{~nm}$. The corresponding refractive indices are chosen as $n_{1}=1.05$ and $n_{2}=1.012$ (arbitrary numbers). The specimen is placed at a distance of $100 \mathrm{~nm}$ from the reference plane (see Fig.1). The corresponding freespace refractive index is $n_{0}=1$. A broadband light source operating in the $[700 \mathrm{~nm}, 900 \mathrm{~nm}]$ wavelength range is chosen. The simulation of this physical set-up yields a parametric model of the form (3) with $L=2,\left\{a_{0}=-0.0244, a_{1}=\right.$ $\left.0.0184, a_{2}=0.0060\right\}$ and $\left\{b_{0}=0.0667 \times 10^{-14}, 0.1500 \times\right.$ 

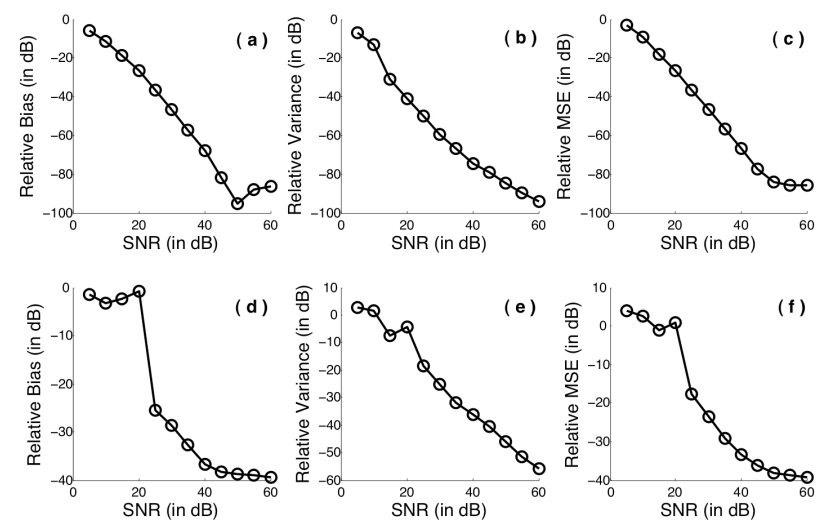

Fig. 2. The relative bias, variance and mean square error, all in $\mathrm{dB}$, as a function of the SNR (dB). The upper row of plots correspond to $b_{2}$ and the lower row of plots corresponds to $a_{2}$. Each data point is obtained by averaging over 100 realizations. We note that the estimators exhibit strong consistency with respect to increase in the SNR.

$\left.10^{-14}, 0.2120 \times 10^{-14}\right\}$. We sample $M(\lambda)$ uniformly on the $1 / \lambda$ axis to yield uniformly-spaced data on the frequency axis. We used a sampling rate which is 10 times the wavelength spread of the source. $S(\nu)$ is assumed to be constant over the operating wavelength range. $N(\nu)$ is generated by a pseudo-random number generator according to a uniform distribution. We used 64 data samples for the experiment. We varied the signal-to-noise ratio (SNR) from $5 \mathrm{~dB}$ to $60 \mathrm{~dB}$ in steps of $5 \mathrm{~dB}$. We generated 100 Monte-Carlo realizations of the noisy signal for each value of the SNR and applied the algorithm (Sec.5) to estimate the parameters of the exponentials. The bias, variance and mean square error (MSE) are computed for one set of parameters $a_{2}$ and $b_{2}$. Since the parameter values themselves are small, we perform normalization of the estimator with respect to the actual value. We plot these quantities as a function of the SNR in Fig.2. From the performance plots, we observe that the estimator displays strong consistency with respect to increase in SNR i.e., there is a decrease in variance with an increase in SNR. The accuracy of estimation is also good; for example, at $25 \mathrm{~dB}$ SNR, the relative mean square error in the estimate of $b_{2}$ is about $-40 \mathrm{~dB}$. This means that the error in the estimate of $b_{2}$ is $0.01 \%$ of the actual value of $b_{2}$. The SNR in a controlled experiment is higher than $25 \mathrm{~dB}$. Thus, we can be assured of more accurate estimates. The small fluctuations in the performance curves are due to the finite number of Monte-Carlo realizations.

\section{CONCLUSIONS}

We have proposed a novel and efficient model-based highresolution technique for the fundamental inverse problem in frequency-domain optical coherence tomography. With minimum number of samples, the technique provides a quantitative description of the layered specimen. The technique is also robust to additive noise and provides accurate estimates. Apart from biomedical applications such as cornea-imaging, the technique may be useful in other areas such as optical fiber layer characterization and monitoring during the manufacturing process.

\section{Acknowledgments}

We thank Prof. Rainer Leitgeb, Prof. Theo Lasser, Adrian Bachmann and Martin Villiger of IOA, EPFL, Switzerland, for helpful discussion on FDOCT. This work was supported in part by the Center for Biomedical Imaging (CIBM) of the Geneva-Lausanne Universities and the EPFL; and the foundations - Leenaards and Louis-Jeantet.

\section{REFERENCES}

[1] R. Leitgeb, C. K. Hitzenberger and A. F. Fercher, "Performance of Fourier domain vs. time domain optical coherence tomography", Vol.11, No. 8, Optics Express, pp. 889-894, 21 April 2003.

[2] A. F. Fercher, C. K. Hitzenberger, G. Kamp, S. Y. El-Zaiat, "Measurement of intraocular distances by backscattering spectral interferometry", Opt. Comm., 117, pp. 43-48, 1995.

[3] G. Hausler, M. W. Lindner, "Coherence radar and spectral radar - new tools for dermatological analysis", Jl. Biomed. Opt., 3, pp.21-31, 1998.

[4] M. Wojtkowski, R. Leitgeb, A. Kowalczyk, T. Bajraszewski, A. F. Fercher, "In-vivo human retinal imaging by fourier domain optical coherence tomography", Jl. Biomed. Opt., 7, pp.457-463, 2002.

[5] B.E.Bouma and G.J. Tearney (Ed.), Handbook of Optical Coherence Tomography, Marcel Dekker, Inc., 2002.

[6] A. Ozcan, M. J. F. Digonnet, G. S. Kino, "Minimumphase-function-based processing in frequency-domain optical coherence tomography systems", Vol. 23, No. 7, J. Opt. Soc. Am. (A), July 2006.

[7] P. Stoica and R. Moses, Introduction to Spectral Analysis, Prentice Hall, 2000.

[8] M. Vetterli, P. Marziliano, T. Blu,"Sampling signals with finite rate of innovation," IEEE Trans. on Sig. Proc., Vol. 50, No. 6, pp. 1417-1428, June 2002. 